\title{
SURFACE EFFECTS ON NEUTRON SCATTERING IN HELIUM FILMS
}

\author{
B. LAMBERT, D. SALIN
}

Laboratoire d'Ultrasons (*), Université Pierre-et-Marie-Curie, Tour 13, 4, place Jussieu, 75230 Paris Cedex 05, France

\section{J. JOFFRIN and R. SCHERM}

Institut Laüe-Langevin, 156 X, Centre de tri, 38042 Grenoble Cedex, France

(Reçu le 23 juin 1977, accepté le 19 août 1977)

\begin{abstract}
Résumé. - Nous décrivons une expérience de diffusion de neutrons par des films minces d'hélium déposés sur du graphite exfolié. Les résultats s'expliquent en tenant compte des effets dus aux forces de Van der Waals entre le substrat et les atomes d'hélium.
\end{abstract}

\begin{abstract}
We report a neutron scattering experiment on thin helium films deposited on exfoliated graphite. The results can be explained by taking into account the effects of Van der Waals forces between the substrate and the helium atoms.
\end{abstract}

The study of the properties of helium in restricted geometries has developed extensively in the last few years. In porous vycor, a multiconnected array of narrow channels, the temperature of the superfluid transition is considerably decreased [1]. On the other hand, exfoliated recompressed graphite offers a well characterized plane substrate with a fairly large specific area on which thin helium films can be condensed [2].

Very little is yet known about the magnitude of the effect of the restricted geometry on the microscopic properties of superfluid $\mathrm{He}$. Measurements of the superfluid density $\rho_{\mathrm{s}} / \rho$ by the oscillating disk method [3] or by observation of third sound [4], and persistent currents [5] gave rise to speculations that the roton energy might be decreased in the thin film [6]. The existence of an additional excitation, the surface roton has been postulated to explain thermodynamic data [6].

The study of the elementary excitations of $\mathrm{He}$ in restricted geometries was suggested several years ago [3]. It was only recently that the first neutron scattering results became available [7]. In the following, we report a similar and independent neutron scattering study of the elementary excitations in $\mathrm{He}$ in thin film geometry; we try to explain these results by a model which takes into account the inhomogeneities in the film.

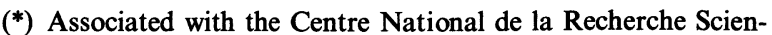
tifique.
1. Experimental. - Helium 4 films were deposited on exfoliated and recompressed graphite (Papyex from Carbone-Lorraine). The specific area of this substrate is about $20 \mathrm{~m}^{2} / \mathrm{g}$. Our sample was $50 \times 70 \times 8 \mathrm{~mm}^{3}$, corresponding to a total surface of approximately $1000 \mathrm{~m}^{2}$. The basal planes of graphite are roughly oriented exhibiting a mosaic distribution with $23^{\circ}$ (full width at middle height). The thickness of the helium film varied from approximately six layers (one atomic layers : $a_{0}=3.6 \AA$ ) to the full distance between the planes of the substrate; in the second case, the properties are practically identical to those of bulk helium. The layer thickness was determined from the total amount of adsorbed

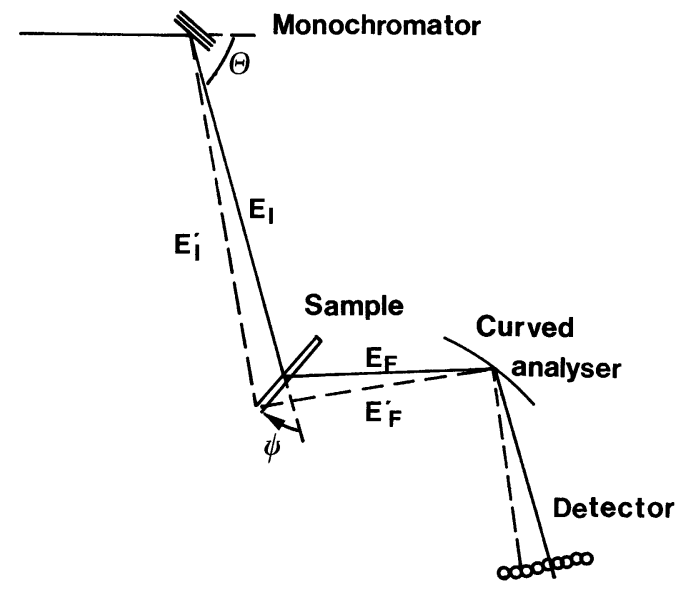

FIG. 1. - Schematic diagram of the spectrometer $\mathrm{IN}_{3}$ in the configuration used in the experiment. 
gas under the assumption that the specific mass of the first three layers were $0.125,0.081$ and $0.077 \mathrm{~g} / \mathrm{cm}^{3}[8]$.

The experiment was performed at the IN3 three axis spectrometer at the I.L.L., Grenoble. A graphite 002 monochromator with a Be filter was used to select an incident energy of $0.98 \mathrm{THz}$. In contrast to the classical set-up, no Soller collimator restricted the angular divergence. The analyser was also made of graphite 002 which was shaped in the form of a cylindrical surface of $1.5 \mathrm{~m}$ radius in the horizontal plane, thus focussing one sharp energy only from a point sample onto the detector [10].

In the actual case, however, the sample is by no means a point but rather an extended plate $5 \mathrm{~cm}$ wide and $0.8 \mathrm{~cm}$ thick. As illustrated in figure 1 the angular orientation $\psi$ of the sample can be chosen in such a way that

$$
\hbar \omega=E_{\mathrm{I}}-E_{\mathrm{F}}=E_{\mathrm{I}}^{\prime}-E_{\mathrm{F}}^{\prime} .
$$

This applies equally well for beams hitting different points along the sample. In this way the effective solid angle and hence the intensity could be increased by a factor 5 without any loss of resolution.

Constant $Q$ scans have been recorded at wave vector transfer $Q=1.92 \AA^{-1}$ which corresponds to the roton minimum, and at $Q=2 \AA^{-1}$. The precision on $Q$ was between $10^{-2} \AA^{-1}$ and $2 \times 10^{-2} \AA^{-1}$. The $Q$-vector was nearly parallel to the substrate basal plane. The temperature was held at $1.4 \mathrm{~K}$; thus the natural roton line width is still much smaller than the instrumental resolution of $30 \mathrm{GHz}$.

2. Results. - Figure 2 shows the neutron groups taken at $Q=1.92 \AA^{-1}$ at different coverages. The background, essentially the tail of the elastic scattering of the substrate, has been measured in absence of helium and then substracted.

Figure 3 shows the integrated intensity of the roton line as a function of coverage; the integration extended from $140 \mathrm{GHz}$ to $230 \mathrm{GHz}$ or from $145 \mathrm{GHz}$ to $215 \mathrm{GHz}$ without changing the figure very much. This method was repeated for two wave vector transfers $Q=1.92 \AA^{-1}$ and $Q=2.0 \AA^{-1}$.

By decreasing progressively the coverage we noted the following facts :

1. There is a slight displacement (about $5 \mathrm{GHz}$ ) of the maximum towards low energies.

2 . The roton peak becomes more and more asymmetrically broadened. These two observations are more obvious if we look at the experimental results in figure 4 where the maxima of the curves are normalized.

3. Extrapolated to zero intensity, the two lines on figure 3 do not intersect. However the ordinate may be shifted downward : this corresponds to a slight modification of the background. By pushing downward the origin of the intensity to this point where the two lines intersect, we find an abscissa of the order

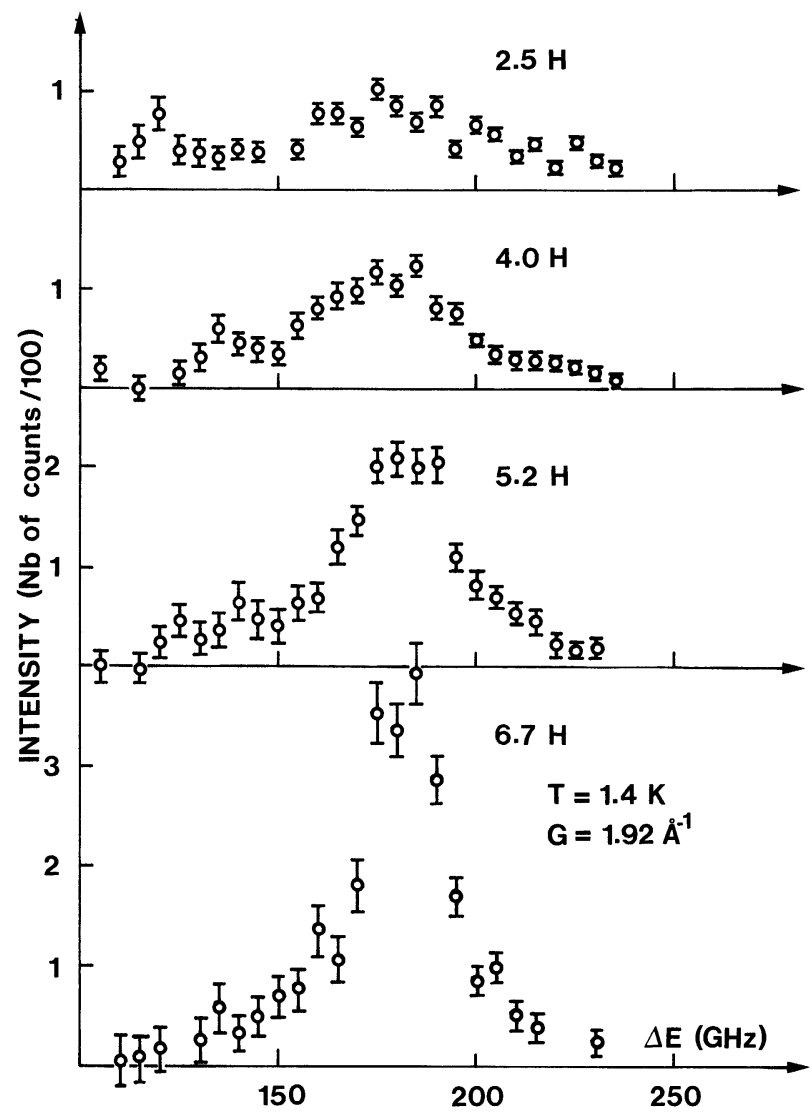

FIG. 2. - Representative spectra from helium on Papyex substrate at $Q=1.92 \AA^{-1}$. The total number of atomic layers has been calculated for an area of $20 \mathrm{~m}^{2} / \mathrm{g}$. The value of $H$ is estimated to be $2.5 a_{0}$.

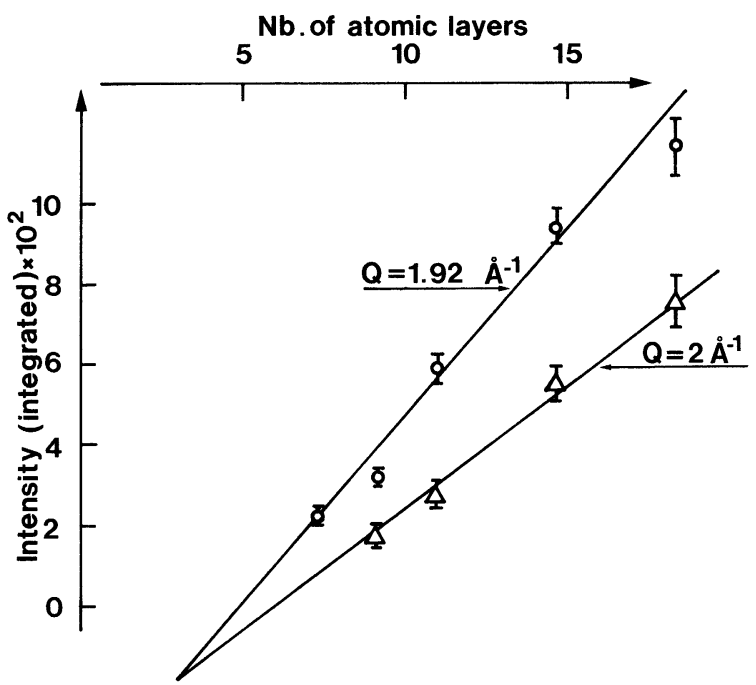

FIG. 3. - These curves give the integrated signal intensity as a function of coverage; the integration has been effected from $140 \mathrm{GHz}$ to $230 \mathrm{GHz}$. The spots correspond to $Q=1.92 \AA^{-1}$; the triangles correspond to $Q=2.0 \AA^{-1}$. They intersect for a thickness roughly equal to $H \simeq 2.5$ layers. The ordinate has been corrected to take into account the background.

of 2.5 atomic layers. It corresponds to the thickness $H$ of the solid helium layer close to the substrate; this layer does not participate in the scattering. 


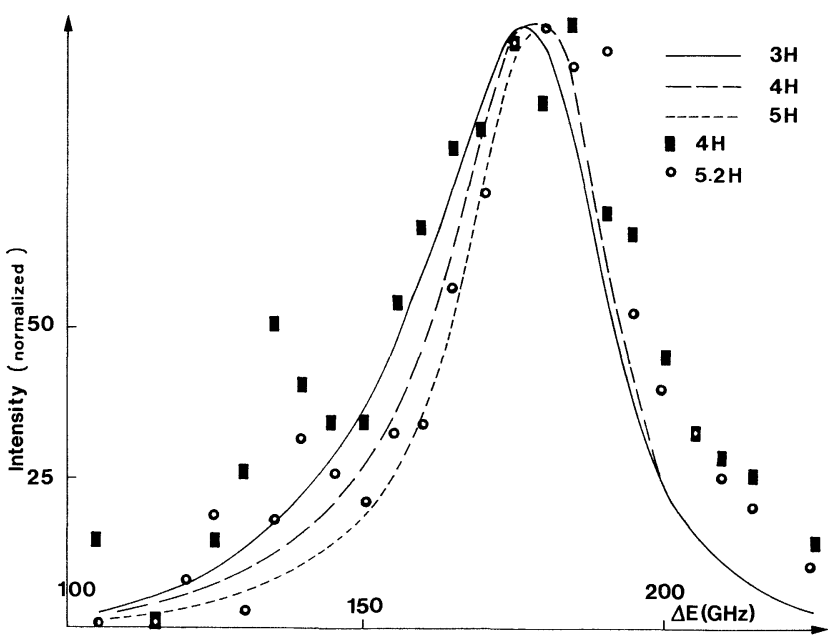

Fig. 4. - The maxima of the curves have been normalized to the same value. The lines correspond to calculated values (full line $3 H$, broken line $4 H$ and dashed line $5 H$ ) corresponding to $\Delta_{0}=180 \mathrm{GHz}, \eta=8.7 \times 10^{-3} \mathrm{~atm}^{-1}, D=15 \mathrm{GHz}, \Gamma_{0}=11 \mathrm{GHz}$ et $\Gamma_{\text {app }}=30 \mathrm{GHz}$.

3. Interpretation. - Van der Waals forces between the substrate and helium atoms play an essential role in stabilizing the film. They also induce inhomogeneities in the static properties; the first layers are more compressed than the external ones; the first two layers are probably solid, and the others progressively acquire the characteristics of bulk helium at saturated vapor pressure as the distance from the substrate increases.

In fact treating the adsorbed helium as a continuum and approximating the Van der Waals potential at the graphite substrate by

$$
\mu_{\mathrm{V} . \mathrm{d} . \mathrm{w} .}=-\frac{\alpha}{z^{3}}
$$

with $\alpha=65 \mathrm{~K}$ (layers) ${ }^{3}$, the thickness $H$ of the solid layer turns out to be [9] :

$$
H=\sqrt[3]{\frac{\rho \alpha}{m P_{\mathrm{s}-1}\left(T_{0}\right)}} \simeq 2.5 a_{0}=9 \AA
$$

in the limit of large film thickness. Here $P_{\mathrm{s}-1}$ denotes the equilibrium pressure between liquid and solid bulk He, and $\rho$ is the specific mass at $T_{0}$ and $m$ is the mass of a $\mathrm{He}$ atom. Finally the pressure within a film of thickness $d$ is given by [9] :

$$
P(z)=P_{\mathrm{s}-1}\left(T_{0}\right)\left[\left(\frac{H}{z}\right)^{3}-\left(\frac{H}{d}\right)^{3}\right]+P_{0}
$$

with $P_{0}$ being the S.V.P. at $T_{0}$.

Knowing the pressure $P(z)$ within the film we reconstruct the shape of the roton line by superposing contributions to the spectrum coming from infinitely thin layers of liquid $\mathrm{He}$ exhibiting bulk properties at different pressures.
Both the roton gap $\Delta(P)$ and linewidth $\Gamma(P)$ (full width at half maximum) are given from [11] by

$$
\begin{aligned}
\Delta & =\Delta_{0}(1-\eta P) \\
\Gamma & =\Gamma_{0}(1-\lambda P)=\Gamma_{0}\left(1+\frac{\Delta_{0}-\Delta}{D}\right)
\end{aligned}
$$

with

$$
\begin{aligned}
\Delta_{0} & =180 \mathrm{GHz} \\
\eta & =8.7 \times 10^{-3} \mathrm{~atm}^{-1} \\
D & =15 \mathrm{GHz} \\
\Gamma_{0} & =11 \mathrm{GHz} \\
P_{s-1}\left(T_{0}\right) & =30 \mathrm{~atm} .
\end{aligned}
$$

The resulting line shape has then been convoluted with a Gaussian of $\Gamma_{\text {app }}=30 \mathrm{GHz}$ F.W.H.M., thus including the experimental resolution.

The results of the calculation show a broadening of the line at lower energies and a shift of the maximum (towards a lower value) with decreasing helium coverage. Figure 4 shows the line shape calculated for three different thichnesses $d(3 H, 4 H, 5 H)$, and corresponding experimental data. The conclusions which can be drawn are : the lifetime of the roton excitations in films is not severely decreased in comparison with the bulk helium; no restricted geometry effects have to be invoked to explain the shift in energy of the rotons, since this can be explained by taking into account the inhomogeneities induced by the Van der Waals forces.

The discrepancy between the experimental results and the calculated curves on the high energy side of the lines has been recently observed by the Brookhaven group [7]; it has been interpreted as being primarely due to the wave vector component perpendicular to the substrate.

The last feature of the spectrum is the behaviour of the integrated scattered intensity as a function of coverage. In figure 3 the extrapolated curve intersects the abscissa at a non zero value; it reflects the influence of the solid layer, the thickness of which turns out to be $(2.5 \pm 0.5)$ layers; $K$. Carneiro et al. have found the same value [7]. This value was previously estimated to be 2.3 layers in a quite different experiment [9].

T. C. Padmore [12] postulated the existence of surface rotons at an energy considerably lower than the bulk roton energy. There is the suggestion of a slight bump in our data at about $0.130 \mathrm{GHz}$; such a value corresponds to the energy peak of surface rotons predicted by the theory [12]. Certainly the statistical accuracy of the data does not permit us to assert the existence of surface rotons. Further investigations on this particular problem are underway.

In conclusion, we emphasize that the model of inhomogeneities satisfactorily describes the spectrum of rotons in thin films. It should be used in further interpretations of data on thin films; it even ought to form the basis of calculations treating thermodynamic properties. 


\section{References}

[1] Brewer, D. F., Champeney, D. C. and Mendelsshon, K., Cryogenics 1 (1960) 1.

[2] Bretz, M., Phys. Rev. Lett. 38 (1977) 501.

[3] Cheeke, J., Hebral, B. and Richard, J., J. Low Temp. Phys. 12 (1973) 359.

[4] Brewer, D., Symonds, A. and Thomson, A., Phys. Rev. Lett. 15 (1965) 182.

[5] Kiewet, C., Hall, H. and RePPy, J., Phys. Rev. Lett. 35 (1973) 1286.

[6] Chester, M. and Eytel, L., Phys. Rev. B 13 (1976) 1069.

[7] Carneiro, K., Ellenson, W., Passel, L., McTague, J. and TAUB, H., Phys. Rev. Lett. 37 (1976) 1695.
[8] Bretz, M., Phys. Rev. Lett. 31 (1973) 1447.

[9] Lambert, B., Perzynski, R., Salin, D. and Joffrin, J., J. Low Temp. Phys. 28 (1977) 359.

[10] Scherm, R., Schedler, E., WAGner, V., Dolling, G., RitTER, R. and TeUCHERT, W., Nucl. Instrum. Methods (1977).

[11] Dietrich, O. W., Graf, E. H., Huang, C. H. and Passell, L., Phys. Rev. A 5 (1972) 1377.

[12] Padmore, T. C., Phys. Rev. Lett. 32 (1974) 826. 